\title{
Decision making under incompleteness based on soft set theory *
}

\author{
José Carlos R. Alcantud ${ }^{1,2 \star \star 0000-0002-4533-9281 ~ a n d ~}$ \\ Gustavo Santos-García ${ }^{2}$ 0000-0001-6609-5493 \\ 1 BORDA Research Unit, University of Salamanca, Spain \\ 2 Facultad de Economía y Empresa and Multidisciplinary Institute of Enterprise \\ (IME), University of Salamanca, Spain \\ $\{$ jcr, santos $\} @$ usal.es \\ http://diarium.usal.es/jcr http://diarium.usal.es/santos
}

\begin{abstract}
Decision making with complete and accurate information is ideal but infrequent. Unfortunately, in most cases the available information is vague, imprecise, uncertain or unknown. The theory of soft sets provides an appropriate framework for decision making that may be used to deal with uncertain decisions. The aim of this paper is to propose and analyze an effective algorithm for multiple attribute decision-making based on soft set theory in an incomplete information environment, when the distribution of incomplete data is unknown. This procedure provides an accurate solution through a combinatorial study of possible cases in the unknown data. Our theoretical development is complemented by practical examples that show the feasibility and implementability of this algorithm. Moreover, we review recent research on decision making from the standpoint of the theory of soft sets under incomplete information.
\end{abstract}

Keywords: Soft sets; Decision making; Incomplete information; Choice value; Combinatorics.

\section{Introduction}

The aim of this paper is to propose an effective algorithm that facilitates a multiple attribute decision-making based on the theory of soft sets under incomplete information, under the general assumption that the distribution of the incomplete data is unknown. Han et al. [16], Qin et al. [26] and Zou and Xiao [37] laid the foundation of soft-set based decision making under incomplete information. Here we follow a novel and different approach.

Complete and accurate information is ideal for decision making, but this situation is rarely met in practice. In most cases the available information is vague, imprecise, uncertain or unknown.

* The second author is grateful to the Spanish projects TRACES TIN2015-67522C3-3-R and Strongsoft TIN2012-39391-C04-04.

** Corresponding author. Address for correspondence: Facultad de Economía y Empresa. Campus Unamuno. E37007 Salamanca, Spain. 
Motivated by these concerns, Zadeh [35] marked the beginning of fuzzy sets theory. At its core, partial membership allows that imprecise information about more complex situations can be faithfylly represented and correctly handled. Many extensions of fuzzy sets broaden their scope (v., $[4,10]$ for definitions and relationships). Relatedly, Molodtsov initiated the concept of soft set theory [25], whereas Aktaş and Çağman [1], Ali et al. [9], Maji et al. [24], Sezgin and Atagün [29] and Feng et al. [15] are further essential references. Concerning extensions of soft sets, Maji, Biswas and Roy [22] introduced fuzzy soft sets (v., [2,3,8,20] for decision making criteria in this model), and Wang, Li and Chen [30] introduced hesitant fuzzy soft sets. Ma et al. [21] provided a review of decision making methods based on hybrid soft set models.

Maji et al. [23] provides a criteria for selecting an object in a soft set scenario, which consists of maximization of the choice values of the problem. Applications of extended soft set theory include rule mining (Herawan and Deris [18]), data mining processes (Qin et al. [27]), international trade (Xiao et al. [31]), and medical diagnosis (cf., e.g., $[7,11,12,17,34])$. Recent advances in this field include the parameter reduction problem in soft set based decision making (cf., [36]).

Zou and Xiao [37] observed that in the process of collecting data, the practitioner often encounters unknown, missing or inexistent data, which suggests the concept of incomplete soft sets (v., Han et al. [16], Qin et al. [26], Lin et $a l$. [19] and Alcantud and Santos-García [5,6] for additional analyses). Deng and Wang [14] extended this notion to incomplete fuzzy soft sets in order to predict unknown data in fuzzy soft sets. ${ }^{3}$

However, there are situations where there is perfect uncertainty about the real value of missing data, or we are sure that the alternatives or attributes are independent. In those cases we cannot presume that averages, probabilities or any other specific evaluations produce reliable estimations as in previous solutions. To deal with these situations Alcantud and Santos-García [6] presented a completely redesigned approach to soft set based decision making problems under incomplete information. It relies on the classical Laplacian argument of probability theory and consequently it suggests to examine all completed tables arising from the original incomplete table. All these tables are then evaluated as is standard, i.e., by their respective choice values (cf., [23]). The alternatives are ultimately ranked according to the number or proportion of tables where they are choice value maximizers. The computational costs of this procedure are examined in [6]. The conclusion is that with a large number of missing values, the problem cannot be efficiently solved by bruteforce. Hence in [5] these authors propose two modified algorithms that permit to tackle problems where the number of unknown values is larger.

In this paper we produce an exact solution to that problem based on the application of combinatorics. We provide an algorithm that implements the mathematical solution. The computational performance of the algorithm is then compared with prior solutions in the literature.

${ }^{3}$ Although Yang et al. [32] showed some weaknesses of this approach, Deng and Chen [13] successfully resolved these conflicting issues. 
The remainder of this paper is organized as follows. Section 2 briefly states some terminology and definitions from soft set theory. Then we review the relevant antecedents, define the technical notions that we need, and recall the domination sieve for incomplete soft sets. Section 3 shows the steps in the proposed algorithm, as well as a fully developed example that proves the feasibility and implementability of our proposal. Finally, Section 4 concludes the paper.

\section{Definitions and Notation}

\subsection{Definitions: Soft Sets and Incomplete Soft Sets}

In this section, we first introduce the standard definitions for complete and incomplete soft sets. There is a fixed universe $U$ of objects, options or alternatives, and there is a universal set $E$ of parameters, characteristics or attributes.

Definition 1 (Molodtsov [25]). A pair $(F, A)$ is a soft set over $U$ when $A \subseteq E$ and $F: A \longrightarrow \mathcal{P}(U)$, where $\mathcal{P}(U)$ denotes the set of all subsets of $U$.

Definition 2 (Han et al. [16]). A pair $(F, A)$ is an incomplete soft set over $U$ when $A \subseteq E$ and $F: A \longrightarrow\{0,1, *\}^{U}$, where $\{0,1, *\}^{U}$ is the set of all functions from $U$ to $\{0,1, *\}$.

The $*$ symbol in the previous definition represents an unknown data. In other words, if the membership of an element $u$ in the subset of $U$ approximated by $e$ is unknown, then $F(e)(u)=*$. Of course, as in standard soft sets, if an object $u$ is (resp. is not) an element of the subset of $U$ approximated by $e$, then $F(e)(u)=1$ (resp., $F(e)(u)=0$ ). Thus any soft set can be regarded as an incomplete soft set in a natural way.

Henceforth we assume $U=\left\{u_{1}, u_{2}, \ldots u_{N}\right\}$ and $A=\left\{e_{1}, e_{2}, \ldots e_{M}\right\}$. Yao [33] explained that under this finiteness assumption, soft sets can be represented either by matrices or in tabular form. Rows correspond to the options, and columns correspond to the parameters. The same is true for incomplete soft sets. Suppose that $T=\left(t_{i j}\right)_{N \times M}$ is the $N \times M$ matrix associated with the incomplete soft set $(F, A)$. Then element $t_{i j}$ is either one or zero or $*$, depending on whether object $i$ verifies property $j$, does not verify it, or it is unknown whether $i$ verifies property $j$, respectively.

Concerning the choice decision mechanism for soft sets, we agree with existing literature in that choice values should be used. As to incomplete soft sets, our proposal is original in that it does not discard any of the possible filled tables or completed soft sets.

\subsection{Previous literature}

Table 1 summarizes the main previous approaches to our problem. 
Table 1. Summary of research studies about incomplete soft set based decision making. The indexes are named as in the original papers.

\begin{tabular}{ll}
\hline Methodology and references & Indexes \\
\hline Weight-average of all possible choice values of objects. & $d_{i}$, \\
Weights of each choice value given by distribution of the & $d_{i-p}$, \\
other objects. & $c_{i(0)}$, \\
Original approach [37] & $c_{i(1)}$ \\
\hline Data filling based on association between parameters. & $Q_{i}$ \\
Choice: choice values for completed set. & \\
It presumes that objects are cross-related. & \\
Inspired by above approach [26] & \\
\hline
\end{tabular}

Elicitation criterions for incomplete soft sets generated by restricted intersection.

This problem is related although different [16]

Elimination of dominated options.

Random sam- $s_{i}^{2}$
ple, thus results
depend on sam-
ple $[5]^{\dagger}$

Choice mechanism: choice values.

Laplacian argument: equal probability to all completed tables. Suitable when there is no guarantee that objects are related to each other.

Brute force, thus $s_{i}$ computationally costly $[6]$

\subsection{Notation and Fundamentals of our Algorithm}

From the input matrix $T$ we calculate the number of ones and unknown values for every object in the soft set. These are fundamental elements for the analysis of the optimal solution in a fully uncertain environment.

Let $v^{1}$ be the vector of numbers of 1's (ones values) by rows in $T$, i.e., $v^{1}=\left(v_{1}^{1}, \ldots v_{N}^{1}\right)$, where $v_{i}^{1}$ is the number of ones in object $i$. Observe that if $c_{i(0)}$ is $i$ 's choice value if all missing data are replaced with 0 , then $v_{i}^{1}=c_{i(0)}=$ $\left|\left\{e_{j} \in A: F\left(e_{j}\right)\left(u_{i}\right)=1\right\}\right|$.

The maximum value in vector $v^{1}$ is $c_{0}=\max \left\{c_{i(0)}: i=1, \ldots, N\right\}=$ $\max \left\{v_{i}^{1}: i=1, \ldots, N\right\}$.

Let $v^{*}$ be the vector of numbers of $*$ 's (unknown values) by rows in $T$, i.e., $v^{*}=\left(v_{1}^{*}, \ldots v_{N}^{*}\right)$, where $v_{i}^{*}$ is the number of unknown values in object $i$. This means $v_{i}^{*}=\left|\left\{e_{j} \in A: F\left(e_{j}\right)\left(u_{i}\right)=*\right\}\right|$. We let $m_{*}$ denote the maximum value in vector $v^{*}$, i.e., $m_{*}=\max \left\{v_{i}^{*}: i=1, \ldots, N\right\}$.

Observe that if $c_{i(1)}$ is $i$ 's choice value when all missing data are replaced with 1 , then $c_{i(1)}=c_{i(0)}+v_{i}^{*}$ for each $i$. 
The number of unknown values is $M^{*}=\sum_{i=1}^{N} v_{i}^{*}$. A simple combinatorial analysis shows that the number of combinations of unknown choice values is:

$$
M^{c}=\prod_{i=1}^{N}\left(v_{i}^{*}+1\right)
$$

Example 1 below illustrates these notions.

\subsection{Domination Sieve}

In order to gain efficiency, our algorithm performs a pre-screening by removing the objects whose choice values can never be maximal. Put shortly, we eliminate dominated alternatives defined as follows:

Definition 3 (Alcantud and Santos-García [6]). Let $(F, A)$ be an incomplete soft set over $U$. Option $i$ dominates option $k$ if and only if $c_{k(1)}<c_{i(0)}$.

Hence in order to check if an option $i$ dominates an option $k$ one needs to verify if $c_{k(0)}+v_{k}^{*}<c_{i(0)}$. Intuitively, no matter how we complete the soft set, option $i$ has a choice value that is strictly higher than the choice value of option $j$. Therefore dominated options should be rejected in any choice-valued approach.

In order to simplify our problem we compute the maximum value $c_{0}=3$ of the choice values $v_{i}^{1}=c_{i(0)}$ for every $i$. If $c_{0}$ is greater than the choice value $c_{k(1)}$ of object $u_{k}$, then object $u_{k}$ can be dropped from the initial matrix because its choice value can never be maximum in any posible completed soft set. In this way, by reducing the number of rows in the matrix form of an incomplete soft set, there are less missing data, which reduces runtime and improves final results.

After dominated options are sieved out, we apply the remaining steps to the new trimmed matrix. To simplify the notation, this reduced matrix is also called $T$. Its objects and features will be reappointed $N$ (also number of rows of $T$ ) and $M$ (also number of columns of $T$ ), respectively.

Example 1. Let $T=\left(t_{i j}\right)$ be the following $5 \times 4$ initial matrix, which represents an incomplete soft set over a universe of 5 objects with 4 relevant characteristics for evaluation of the alternatives:

$$
T=\left(\begin{array}{cccc}
1 & 1 & 1 & 0 \\
1 & 1 & * & 0 \\
1 & * & * & 0 \\
1 & * & 0 & 0 \\
0 & 0 & 0 & 0
\end{array}\right)
$$

It is easy to compute that $v^{1}=(3,2,1,1,0), v^{*}=(0,1,2,1,0)$ therefore $m_{1}=3, m_{*}=2, M^{*}=4$, and $M^{c}=12$.

We calculate the maximum value $c_{0}$ of all choice values $c_{i(0)}$. Observe that $c_{4(0)}+v_{4}^{*}=1+1<c_{1(0)}=c_{0}=3$ and $c_{5(0)}+v_{5}^{*}=0+0<c_{1(0)}=c_{0}=3$. Thus $c_{0}$ is unattainable for the choice values $c_{i(1)}$ of objects $u_{4}$ and $u_{5}$, and these objects 
Table 2. Tabular representation of the soft set in Example 1, and noteworthy associated indices.

\begin{tabular}{|c|cccc|rrr|}
\hline & $e_{1}$ & $e_{2}$ & $e_{3}$ & $e_{4}$ & $v_{i}^{1}=c_{i(0)}$ & $c_{i(1)}$ & $v_{i}^{*}$ \\
\hline$u_{1}$ & 1 & 1 & 1 & 0 & $c_{0}=\underline{3}$ & 3 & 0 \\
$u_{2}$ & 1 & 1 & $*$ & 0 & 2 & 3 & 1 \\
$u_{3}$ & 1 & $*$ & $*$ & 0 & 1 & 3 & 2 \\
$\left(u_{4}\right)$ & 1 & $*$ & 0 & 0 & 1 & $\underline{2}$ & 1 \\
$\underline{\left(u_{5}\right)}$ & 0 & 0 & 0 & 0 & 0 & $\underline{0}$ & 0 \\
\hline
\end{tabular}

can be safely removed from the initial matrix (see Table 2, where the relevant items are underlined). Observe that no matter how the table is completed, the choice values of options $u_{4}$ and $u_{5}$ will be smaller than the choice value of $u_{1}$.

After removing objects $u_{4}$ and $u_{5}$, the new sieved matrix is:

$$
T \text { (sieved })=\left(\begin{array}{cccc}
1 & 1 & 1 & 0 \\
1 & 1 & * & 0 \\
1 & * & * & 0
\end{array}\right)
$$

For the new trimmed matrix the vector of numbers of one values $v_{1}$ and the vector of numbers of unknown values $v^{*}$ are:

$$
v^{1}=(3,2,1) ; \quad v^{*}=(0,1,2)
$$

which can be drawn from the corresponding columns in Table 2. And now it is immediate to compute $m_{1}=3, m_{*}=2$.

Moreover $M^{*}=3$ (number of unknown values) and $M^{c}=6$ (number of combinations of unknown choice values). This means that the four unknown values of the initial matrix $T$ become three values for the new sieve matrix $T$, while the number of feasible states (in terms of configurations of choice values) is halved. These states are:

(1) the choice values can be 3 for option $u_{1}, 2$ for $u_{2}$ and 1 for $u_{3}$. This fact happens in exactly one completed table.

(2) the choice values can be 3 for option $u_{1}, 2$ for $u_{2}$ and 2 for $u_{3}$. This fact happens in exactly two completed tables.

(3) the choice values can be 3 for option $u_{1}, 2$ for $u_{2}$ and 3 for $u_{3}$. This fact happens in exactly one completed table.

(4) the choice values can be 3 for option $u_{1}, 3$ for $u_{2}$ and 1 for $u_{3}$. This fact happens in exactly one completed table.

(5) the choice values can be 3 for option $u_{1}, 3$ for $u_{2}$ and 2 for $u_{3}$. This fact happens in exactly two completed tables.

(6) the choice values can be 3 for option $u_{1}, 3$ for $u_{2}$ and 3 for $u_{3}$. This fact happens in exactly one completed table.

We can represent the completed soft sets for cases (1), (2) and (3) as follows: 


\begin{tabular}{|l||cccc||cccc|cccc||cccc|}
\hline & $e_{1}$ & $e_{2}$ & $e_{3}$ & $e_{4}$ & $e_{1}$ & $e_{2}$ & $e_{3}$ & $e_{4}$ & $e_{1}$ & $e_{2}$ & $e_{3}$ & $e_{4}$ & $e_{1}$ & $e_{2}$ & $e_{3}$ & $e_{4}$ \\
\hline$u_{1}$ & 1 & 1 & 1 & 0 & 1 & 1 & 1 & 0 & 1 & 1 & 1 & 0 & 1 & 1 & 1 & 0 \\
$u_{2}$ & 1 & 1 & 0 & 0 & 1 & 1 & 0 & 0 & 1 & 1 & 0 & 0 & 1 & 1 & 0 & 0 \\
$u_{3}$ & 1 & 0 & 0 & 0 & 1 & 1 & 0 & 0 & 1 & 0 & 1 & 0 & 1 & 1 & 1 & 0 \\
\hline
\end{tabular}

And the completed soft sets for cases (4), (5) and (6) are represented as

\begin{tabular}{|l||cccc||cccc|cccc||cccc|}
\hline & $e_{1}$ & $e_{2}$ & $e_{3}$ & $e_{4}$ & $e_{1}$ & $e_{2}$ & $e_{3}$ & $e_{4}$ & $e_{1}$ & $e_{2}$ & $e_{3}$ & $e_{4}$ & $e_{1}$ & $e_{2}$ & $e_{3}$ & $e_{4}$ \\
\hline$u_{1}$ & 1 & 1 & 1 & 0 & 1 & 1 & 1 & 0 & 1 & 1 & 1 & 0 & 1 & 1 & 1 & 0 \\
$u_{2}$ & 1 & 1 & 1 & 0 & 1 & 1 & 1 & 0 & 1 & 1 & 1 & 0 & 1 & 1 & 1 & 0 \\
$u_{3}$ & 1 & 0 & 0 & 0 & 1 & 1 & 0 & 0 & 1 & 0 & 1 & 0 & 1 & 1 & 1 & 0 \\
\hline
\end{tabular}

\section{Algorithm: Incomplete Soft Sets for Decision Making Problem}

\subsection{The Main Elements of the Algorithm}

Our algorithm computes the number of tables that produce every possible configuration of choice values. Under the Laplacian assumption that they are equally probable, it chooses the alternative that is best in the highest proportion of completed soft sets. However our algorithm does not compute them explicitly as in Example 1, but instead we use a combinatorial analysis in order to define several auxiliary matrices corresponding to possible values and probabilities for each of the objects that we actually use. Let $P V_{1}$ (resp., $\left.P P V_{1}\right)$ be the $\left(N \times\left(m_{*}+1\right)\right.$ )matrices of potential values (resp., proportion by rows of potential values) for each object. For computational purposes, the rows with a number of elements less than $m_{*}+1$ are filled up with zero values. Its elements are calculated according to the following formulas:

$$
P V_{1}(i, j)=v_{i}^{1}+j-1 ; \quad P P V_{1}(i, j)=\frac{\left(\begin{array}{c}
v_{i}^{*} \\
j
\end{array}\right)}{2^{v_{i}^{*}}},
$$

where $i$ varies from 1 to $N$ and $j$ varies from 1 to $v_{i}^{*}+1 .{ }^{4}$ These matrices are shown in Example 2.

Example 2. According to the data of Example 1, now we calculate matrices $P V_{1}$ and $P P V_{1}$ :

$$
P V_{1}=\left(\begin{array}{ccc}
3 & 0 & 0 \\
2 & 3 & 0 \\
1 & 2 & 3
\end{array}\right), \quad P P V_{1}=\left(\begin{array}{ccc}
1.00 & 0 & 0 \\
0.50 & 0.50 & 0 \\
0.25 & 0.50 & 0.25
\end{array}\right)
$$

Hence for example, the fact $P V_{1}(1,1)=3$ and $P P V_{1}(1,1)=1$ means that in $100 \%$ of the 8 filled tables, the choice value of $u_{1}$ is 3 . The fact $P V_{1}(2,2)=3$

\footnotetext{
${ }^{4}$ As usual, $\left(\begin{array}{l}n \\ k\end{array}\right)$ or " $n$ choose $k$ " returns the binomial coefficient, i.e., the number of combinations of $n$ items taken $k$ at a time, defined as $\frac{n !}{(n-k) ! k !}$.
} 
and $P P V_{1}(2,2)=0.5$ means that in $50 \%$ of the 8 filled tables, the choice value of $u_{2}$ is 3 . And $P V_{1}(3,2)=2$ and $P P V_{1}(3,2)=0.5$ means that in $50 \%$ of all filled tables, the choice value of $u_{3}$ is 2 .

It is well-known that if two events $A$ and $B$ are independent then their joint probability equals the product of their probabilities, i.e., $\mathrm{P}(A \cap B)=\mathrm{P}(A) \mathrm{P}(B)$. Because choice values are independent events, with probability $1 \cdot 0.5 \cdot 0.5=0.25$ the choice values of $u_{1}, u_{2}$ and $u_{3}$ are 3,3 , and 2 , respectively. And in that case, both $u_{1}$ and $u_{2}$ are optimal because they are choice value maximizers.

After calculating the auxiliary matrices $P V_{1}$ and $P P V_{1}$, we analyze all feasible combinations of decision values. As shown in Example 2, we know the probabilities for each of the possible events. Computationally, we build all possible different vectors $C V$ with $N$ rows, in which each element will be a non-zero element of each row in $P V_{1}$ matrix. That is, the element $C V_{i}$ for each object $i$ has a value between $c_{i(0)}$ and $c_{i(0)}+v_{i}^{*}$.

For this particular case, we now calculate the probabilities of occurrences from matrix $P P V_{1}$. The joint probability $X P$ is the product of all the individual probabilities. When the choice value of that particular case is maximal (may be several maximal), we add that probability $X P$ to the decision values matrix $D V$.

We repeat the process for all possible vectors $C V$. The final decision of our Algorithm will consist of the object(s) with greater $D V$ values.

Figure 1 shows a flowchart for the proposal of a decision making procedure under incomplete information that we have described throughout this section.

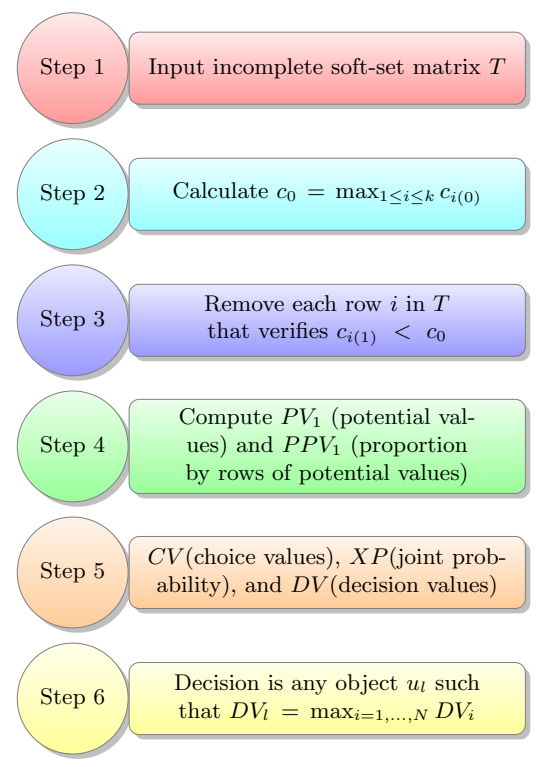

Fig. 1. Step by step procedure for decision making under incompleteness. 
Example 3. According to the data of Example 1 and 2, now we calculate final matrices $C V, X P$, and $D V$. In particular we obtain

$$
D V=\left(\begin{array}{l}
1.00 \\
0.50 \\
0.25
\end{array}\right)
$$

which we interpret as follows. In $100 \%$ of the randomly filled tables, option 1 achieves the maximum choice value. In $50 \%$ of the randomly filled tables, option 2 achieves the maximum choice value. And in $25 \%$ of the randomly filled tables, option 3 achieves the maximum choice value. We recall that ties may happen, which explains why this figures sum up over $100 \%$.

Table 3 contains the elements that produce our solution. In view of our arguments, option 1 should be selected.

Table 3. Combinations of choice values and their respective probabilities in Example 1.

\begin{tabular}{ccccc}
\hline \multicolumn{3}{c}{ Choice value } & Probability & $\begin{array}{c}\text { Optimal } \\
\text { solutions }\end{array}$ \\
\cline { 1 - 3 }$u_{1}$ & $u_{2}$ & $u_{3}$ & & $u_{1}$ \\
3 & 2 & 1 & $1 / 8$ & $u_{1}$ \\
3 & 2 & 2 & $1 / 4$ & $u_{1}, u_{3}$ \\
3 & 2 & 3 & $1 / 8$ & $u_{1}, u_{2}$ \\
3 & 3 & 1 & $1 / 8$ & $u_{1}, u_{2}$ \\
3 & 3 & 2 & $1 / 4$ & $u_{1}, u_{2}, u_{3}$ \\
\hline
\end{tabular}

\subsection{Decision of the Algorithm}

Intuitively, our practical implementation of the ideas above is based on the following arguments. According to Laplace's principle of indifference in probability theory, under complete ignorance we must assume that in all tables $*$ 's are replaced in equiprobable manner with either 0 or 1 . Hence the best we can do is consider in each of these cases that the objects should be selected according to soft-set based decision making procedures. Consequently we should opt for any object that is optimal in the highest proportion of cases with the completed information. The number of possible cases is exponential on the number of unknown values, however we only need to consider completed soft sets which are equivalent for purposes of decision making.

To do this we note that for any object $i$, if it has multiple unknown characteristics that are completed, we are only interested in the number, not in the 
order, of 1's assigned to these unknown values. Thus, the calculation is reduced to a combinatorial analysis in each object.

In accordance with this idea, we endorse the following algorithm for the problems where both $U$ and $A$ are finite:

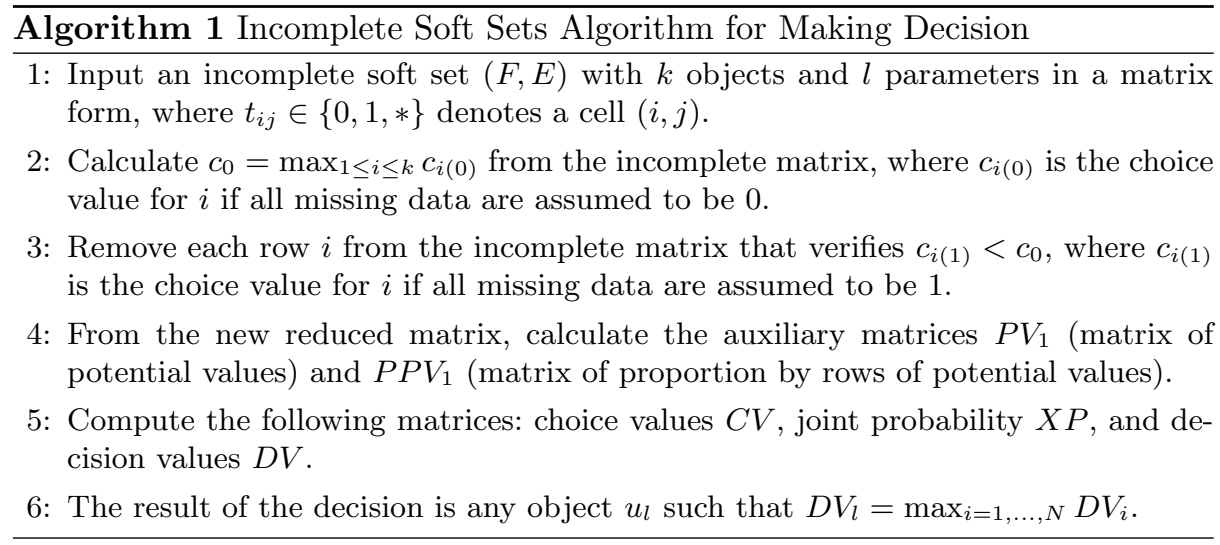

\section{Discusion and conclusions}

The works of Han et al. [16], Qin et al. [26] and Zou and Xiao [37] present interesting approaches to incomplete soft set based decision making. These authors used averages, probabilities or other specific evaluations in order to estimate the real value of missing data in a general way and afterwards, they made a decision based on the complementary data.

In this paper we look at the problem from an altogether different perspective. Rather than filling the incomplete data table (see also Khan et al. [28]), we propose a combinatorial study through all possible filled tables that can be produced from the original incomplete table. Then the alternatives are ranked by the proportion of filled tables where they achieve the highest choice value. In other words, a final indicator for each of the objects by our algorithm is defined as the value of this ratio. And our decision making procedure consists of selecting alternatives that maximize this indicator. A classical Laplacian argument from probability theory justifies our research method. In general there is perfect uncertainty about the real value of missing data. Therefore, we cannot support the idea that other aspects would let us faithfully estimate these unknown values. Under Laplace's principle of indifference, due to our complete ignorance we are entitled to assume that all possible tables where the missing data are replaced with either 0 or 1 are equiprobable.

Our novel approach meets the following targets: (1) We do not need to assume any cross-relations among options. (2) We do not attempt to fill the tables with hypothesized values. (3) We adopt a Laplacian position and make use of combinatorics. (4) A unique, computationally tractable solution is provided. 
An example illustrates the detailed implementation process of our approach and shows evidence of its potential applications in decision-making problems with incomplete information.

In practice, problems where all parameters describing compared options are equally important rarely exist. Our algorithm has been tightly designed for the exclusive purpose of decision making, hence the introduction of weighted parameters would not be trivial and as such deserves a separate analysis. Domination sieve is simple to read in that instance, though.

\section{References}

1. Aktaş, H., Çă̆man, N.: Soft sets and soft groups. Inf Sci 177, 2726-35 (2007)

2. Alcantud, J.C.R.: Fuzzy soft set decision making algorithms: some clarifications and reinterpretations. In: Luaces, O., Gámez, J., Barrenechea, E., Troncoso, A., Galar, M., Quintián, H., Corchado, E. (eds.) 17th Conference of the Spanish Association for Artificial Intelligence. LNAI, vol. 9868, pp. 479-488. Springer (2016)

3. Alcantud, J.C.R.: A novel algorithm for fuzzy soft set based decision making from multiobserver input parameter data set. Inform Fusion 29, 142-148 (2016)

4. Alcantud, J.C.R.: Some formal relationships among soft sets, fuzzy sets, and their extensions. Int J Approx Reason 68, 45-53 (2016)

5. Alcantud, J.C.R., Santos-García, G.: Incomplete soft sets: New solutions for decision making problems. In: Bucciarelli, E., Silvestri, M., Rodríguez-González, S. (eds.) Decision Economics, In Commemoration of the Birth Centennial of Herbert A. Simon 1916-2016 (Nobel Prize in Economics 1978) - Distributed Computing and Artificial Intelligence, 13th International Conference. Adv Intel Syst Comput, vol. 475, pp. 9-17. Springer (2016)

6. Alcantud, J.C.R., Santos-García, G.: A new criterion for soft set based decision making problems under incomplete information. Int J Comput Int Sys 10, 394-404 (2017)

7. Alcantud, J.C.R., Santos-García, G., Galilea, E.H.: Glaucoma diagnosis: A soft set based decision making procedure. In: Puerta, J.M., Gámez, J.A., Dorronsoro, B., Barrenechea, E., Troncoso, A., Baruque, B., Galar, M. (eds.) Lect Notes Artif Int. LNCS, vol. 9422, pp. 49-60. Springer (2015)

8. Alcantud, J.C.R., Mathew, T.J.: Separable fuzzy soft sets and decision making with positive and negative attributes. Applied Soft Computing 59(Supplement C), 586-595 (2017)

9. Ali, M.I., Feng, F., Liu, X., Min, W.K., Shabir, M.: On some new operations in soft set theory. Comput Math Appl 57(9), 1547-1553 (2009)

10. Bustince, H., Barrenechea, E., Pagola, M., Fernandez, J., Xu, Z., Bedregal, B., Montero, J., Hagras, H., Herrera, F., De Baets, B.: A historical account of types of fuzzy sets and their relationships. IEEE Trans Fuzzy Syst 24(1), 179-194 (2016)

11. Çelik, Y., Yamak, S.: Fuzzy soft set theory applied to medical diagnosis using fuzzy arithmetic operations. J Inequal Appl 2013(1), 82 (2013)

12. Chetia, B., Das, P.K.: An application of interval valued fuzzy soft sets in medical diagnosis. Int J Contemp Math Sci 38(5), 1887-94 (2010)

13. Deng, T., Chen, Y.: Comments from the author of "An object-parameter approach to predicting unknown data in incomplete fuzzy soft sets" [Appl Math Model 37 (2013) 4139-4146]. Appl Math Model 39(23-24), 7744-7745 (2015) 
14. Deng, T., Wang, X.: An object-parameter approach to predicting unknown data in incomplete fuzzy soft sets. Appl Math Model 37(6), 4139-4146 (2013)

15. Feng, F., Cho, J., Pedrycz, W., Fujita, H., Herawan, T.: Soft set based association rule mining. Knowl-Based Syst 111, 268-282 (2016)

16. Han, B.H., Li, Y., Liu, J., Geng, S., Li, H.: Elicitation criterions for restricted intersection of two incomplete soft sets. Knowl-Based Syst 59, 121-131 (2014)

17. Hassan, N., Sayed, O.R., Khalil, A.M., Ghany, M.A.: Fuzzy soft expert system in prediction of coronary artery disease. International Journal of Fuzzy Systems 19(5), 1546-1559 (2017)

18. Herawan, T., Deris, M.M.: A soft set approach for association rules mining. KnowlBased Syst 24(1), 186-195 (2011)

19. Lin, H., Xiao, Z., Cheng, Y.: New methods for decision making with soft set under incomplete information. J Comput Theor Nanosci 13(2), 1247-1252 (2016)

20. Liu, Z., Qin, K., Pei, Z.: A method for fuzzy soft sets in decision-making based on an ideal solution. Symmetry 9(10), 246 (2017)

21. Ma, X., Liu, Q., Zhan, J.: A survey of decision making methods based on certain hybrid soft set models. Artif Intell Rev pp. 1-24 (2016)

22. Maji, P., Biswas, R., Roy, A.: Fuzzy soft sets. J Fuzzy Math 9, 589-602 (2001)

23. Maji, P., Biswas, R., Roy, A.: An application of soft sets in a decision making problem. Comput Math Appl 44, 1077-83 (2002)

24. Maji, P., Biswas, R., Roy, A.: Soft set theory. Comput Math Appl 45, 555-62 (2003)

25. Molodtsov, D.: Soft set theory - first results. Comput Math Appl 37, 19-31 (1999)

26. Qin, H., Ma, X., Herawan, T., Zain, J.: Data filling approach of soft sets under incomplete information. In: Nguyen, N., Kim, C.G., Janiak, A. (eds.) Intelligent Information and Database Systems, LNCS, vol. 6592, pp. 302-11. Springer (2011)

27. Qin, H., Ma, X., Zain, J.M., Herawan, T.: A novel soft set approach in selecting clustering attribute. Knowl-Based Syst 36, 139-145 (2012)

28. Sadiq Khan, M., Al-Garadi, M.A., Wahab, A.W.A., Herawan, T.: An alternative data filling approach for prediction of missing data in soft sets (ADFIS). SpringerPlus 5(1), 1-20 (2016)

29. Sezgin, A., Atagün, A.O.: On operations of soft sets. Comput Math Appl 61(5), 1457-1467 (2011)

30. Wang, F., Li, X., Chen, X.: Hesitant fuzzy soft set and its applications in multicriteria decision making. J Appl Math article ID 643785 (2014)

31. Xiao, Z., Gong, K., Zou, Y.: A combined forecasting approach based on fuzzy soft sets. J Comput Appl Math 228(1), 326-333 (2009)

32. Yang, Y., Song, J., Peng, X.: Comments on "An object-parameter approach to predicting unknown data in incomplete fuzzy soft sets" [Appl Math Modell 37 (2013) 4139-4146]. Appl Math Model 39(23-24), 7746-7748 (2015)

33. Yao, Y.: A comparative study of fuzzy sets and rough sets. Information Sciences 109, 227-242 (1998)

34. Yuksel, S., Dizman, T., Yildizdan, G., Sert, U.: Application of soft sets to diagnose the prostate cancer risk. J Inequal Appl 2013(1), 229 (2013)

35. Zadeh, L.: Fuzzy sets. Inf Control 8, 338-53 (1965)

36. Zhan, J., Alcantud, J.C.R.: A survey of parameter reduction of soft sets and corresponding algorithms. Artif Intell Rev (2018), to appear, DOI 10.1007/s10462-0179592-0.

37. Zou, Y., Xiao, Z.: Data analysis approaches of soft sets under incomplete information. Knowl-Based Syst 21(8), 941-5 (2008) 